\title{
Fortalecimiento del proceso de enseñanza aprendizaje de la Medicina Familiar y Comunitaria en Iberoamérica
}

\author{
Strengthening the teaching-learning process of Family and Community Medicine in Iberoamerica \\ Fortalecimento do processo de ensino-aprendizagem da Medicina de Família e Comunitária em \\ Ibero-américa
}

Fernando Coppolillo. Facultad de Medicina Universidad de Buenos Aires. Argentina. fernando.coppolillo@gmail.com (Autor correspondiente) Humberto Jure. Facultad de Medicina, Universidad Nacional del Nordeste. Argentina. hjure2000@ hotmail.com Maria Beatriz Ciuffolini. Facultad de Medicina Universidad Nacional de Cordoba. Argentina. mcifiuffolini@ hotmail.com Diana Yuruhán. Universidad Católica Nuestra Señora de la Asunción. Paraguay. dyuruhan@ hotmail.es

\section{Grupo de Trabajo}

Aguilera Luis (España); Aleida Marlen (Honduras); Alvarez Sintes Roberto (Cuba); Araya Zamora Héctor (Costa Rica); Arias Castillo Liliana (Colombia); Astudillo Romero Xavier (Ecuador); Baza Ricardo (Costa Rica); Braida Julio (Uruguay); Buffa Gabriela (Argentina); Cabrera Haydee (México); Casado Vicente Verónica (España); Cascante Gómez Alejandra (Costa Rica); Fernández Larrauri Milagritos (Peru); Fernández Gutiérrez Herrera Raúl (México); Fernández Ortega Miguel Ángel (México); Galván Barrantes Alonso (Peru); González Pantaleón Violeta (Republica Dominicana); González Reyes Carolina. (Chile); Hoock Jennifer (Estados Unidos); Icaza Carlos (Costa Rica); León Jose Luis (Costa Rica); López Nancy (Chile); Luna Tellez Yossadara (México); Mahecha Rivera Lina Maria (Colombia); Martínez Bianchi Viviana (Estados Unidos); Montalvo Johanna (Ecuador); Mussetti Gustavo. (Uruguay); Niz Clara. (Uruguay); Ramirez Gerardo (México); Rodarte Alina (México); Rojas Tenorio Nancy (Costa Rica); Rodríguez Sotomayor Jorge Jesús (Panamá); Roselli Karina (Uruguay); León Salas Jose Luis (Costa Rica); Sánchez Monge Aurora (Costa Rica); Sandoval Gladys (Paraguay); Silvera Cristina (Panamá); Suárez Sergio Ariel (Argentina); Upun Hi Teresa (Guatemala); Valle Jorge Alberto (Honduras); Valverde Juliana (Costa Rica); Vargas Mejía Carmen (Costa Rica); Vargas Patricia (Chile); Villaseca Silva Patricia (Chile); Ysmodes Yvonne (Perú)

\section{Resumen}

El proceso de enseñanza aprendizaje en Medicina Familiar y Comunitaria es analizado desde la perspectiva de las etapas del ciclo profesional: la formación de grado universitario, la especialización en el posgrado y por ultimo la educación permanente en servicio. Para cada una de estas etapas se analizan las dimensiones de los contenidos y compentencias clínicas que se deben desarrollar, los escenarios del proceso enseñanza aprendizaje y las competencias de los docentes para cada una de las mismas. Este análisis permite orientar al fortalecimiento global de los especialistas en medicina familiar y comunitaria con una practica clínica de alta calidad, centrada en la persona, orientada en la familia y basada en la comunidad.
Cómo citar: Coppolillo F, Jure H, Ciuffolini MB, Yuruhán D. Fortalecimiento del proceso de enseñanza aprendizaje de la Medicina Familiar y Comunitaria en Iberoamérica. Rev Bras Med Fam Comunidade. 2016;11(Suppl 2):46-54. http://dx.doi.org/10.5712/rbmfc11(0)1385

\section{Palabras clave:}

Medicina Familiar y Comunitaria Proceso Enseñanza Aprendizaje Docencia universitaria

Especialización

Educación Permanente en Servicio
Financiación:

ninguna declarada.

Aprobación ética:

La investigación fue considerada una investigación sin riesgo. Los autores declaran que los procedimientos seguidos se realizaron de acuerdo a las normas éticas de la Asociación Médica Mundial y la Declaración de Helsinki. Conflicto de intereses: ninguna declarada. Procedencia y revisión por pares: revisado por pares. Recibido el: 15/08/2016. Aceptado el: 15/09/2016. 


\section{Abstract}

The teaching-learning process in Family and Community Medicine is analyzed from the perspective of the professional cycle stages: the formation of university degree, specialization in graduate school and finally the permanent education service. For each of these stages the dimensions of the content and clinical abilities to be developed, the stage of the teaching-learning process and skills of teachers for each of them are analyzed. This analysis allows to guide the overall strengthening of specialists in family and community medicine with clinical practice of high quality, person-centered, family-oriented and community-based.

\section{Resumo}

O processo de ensino-aprendizagem em Medicina de Família e Comunidade é analisado a partir da perspectiva das fases do ciclo profissional: a formação de graduação, especialização em pós-graduação e, finalmente, a educação permanente em serviço. Para cada uma destas etapas se analisam as dimensões do conteúdo e competências clínicas a serem desenvolvidas, os cenários do processo ensino-aprendizagem e as competências dos professores para cada um das mesmas. Esta análise permite orientar o fortalecimento global dos especialistas em medicina de família e comunidade com uma prática clínica de alta qualidade, centrada na pessoa, orientada na família e baseada na comunidade.

\section{Keywords:}

Family and Community

Medicine

Teaching Learning Process

University teaching

Specialization

Permanent Education Service

Palavras-chave:

Medicina de Família

e Comunidade

Processo Ensino Aprendizagem

Ensino Universitário

Especialização

Educação Permanente

em Serviço

"Enseñar no es transferir conocimiento, sino crear los escenarios para su producción y construcción; quien enseña aprende al enseñar, quien aprende enseña al aprender".

Paulo Freire

\section{Introducción}

El fortalecimiento del proceso enseñanza-aprendizaje (PEA) de los especialistas en medicina familiar y comunitaria (MFC) en la Región constituye un objetivo central en los sistemas de salud para garantizar la accesiblidad a cuidados de calidad, centrados en las personas. El desarrollo de competencias clínicas de los médicos de familia debe ser un objetivo central en las políticas de recursos humanos.

Hemos decidido estructurar el documento siguiendo el proceso de formación de un médico de familia desde los estudios de medicina en la Universidad, la especialización y la educación permanente en servicio una vez que se encuentra en ejercicio profesional. Para darle un carácter sistémico, hemos incluido la formación de los docentes, para que puedan adecuarse a los contenidos y procesos que demandan los sistemas actualmente.

\section{Objetivos}

El proposito de este trabajo es contribuir con el fortalecimiento del PEA de la MFC en la Región para garantizar las competencias clínicas y la calidad de la atención en las comunidades.

Los objetivos fueron:

- analizar los escenarios adecuados de formación, las contenidos curriculares en cada una de las etapas de formación y las características de formación de los docentes en MFC;

- evaluar el nivel de desarrollo MFC en la Universidad, la formación de los docentes y los procesos de certificación de la Especialidad en sus países.

\section{Métodos}

Se desarrollaron dimensiones en cada una de las etapas del PEA en MFC a través de la consulta con expertos. Estas dimensiones fueron: 1. La contribución de la medicina familiar en la Universidad 2. Los contenidos para el proceso de enseñanza aprendizaje 3. Los escenarios adecuados para el proceso 4. La formación de los docentes en MFC. Se realizó un cuestionario semi estructurado que fue distribuido a las Sociedades Científicas de Wonca Iberomaricana CIMF. Un total de veinte profesores de MFC completaron el cuestionario, Tres revisores analizaron en 
forma independiente del documento preliminar y tomaron los indicadores claves. En una segunda etapa se realizó un grupo de foco donde se analizó el trabajo previo y se establecieron los indicadores finales.

En una segunda parte del trabajo se solicitó a los docentes participantes de la etapa inicial que calificaran en una escala Lickert calificando del 1 al 10 (donde 1 es ningún desarrollo y 10 gran desarrollo. Las dimensiones estudiadas fueron el desarrollo de la medicina familiar en la Universidad de sus países, la formación de los docentes y los procesos de certificación de la Especialidad. Se calculó la media para cada una de estas dimensiones.

\section{Resultados}

\section{La contribución de la MFC para el desarrollo de Competencias Clínicas en el Pregrado de las Facultades de Medicina}

Desde hace unas décadas, los sistemas educativos del mundo promueven la innovación de los modelos educativos y curriculares y la incorporación de MFC en el pregrado. En este marco, el docente de MFC aparece como responsable de la incorporación efectiva y del eventual éxito de dichas innovaciones, propiciando el cambio en los distintos escenarios de enseñanza. Sin embargo, la incorporación de las propuestas educativas de MFC, requiere una reflexión profunda, que considere entre otros aspectos, las amenazas provenientes de las políticas educativas, el sistema económico, los movimientos sociales, los estilos de administración y las culturas organizacionales de nuestras Universidades sustentadas en su mayoría en el paradigma biomédico y centrada en la atención hospitalaria.

La incorporación de la MFC en el pregrado permite a los alumnos adquirir competencias al respecto de:

- Abordaje integral y continuo de los problemas de salud del paciente sano;

- aplicar el enfoque de riesgo en los padecimientos más frecuentes;

- aprender acerca del manejo de inmunizaciones y de vigilancia epidemiológica;

- educación para la salud;

- abordaje familiar;

- comunicación con el paciente y familia.

Permite a los estudiantes formarse en un aspecto mas antropologico de la medicina, donde conoce y se interesa por los aspectos psicosociales del paciente y no solo por los biológicos.

En el pregrado dentro debe darse énfasis a la Atención Primaria de la Salud (APS), para que los egresados independientemente de la especialidad elegida posteriormente - puedan comprender la lógica de un sistema de salud que no esté centrada en los Hospitales.

La MFC permite ampliar el marco de abordaje clínico; pasar del análisis de la enfermedad al abordaje de la persona como un todo, en el contexto de su familia (quienes compartirán el manejo de la salud de la persona) y de su comunidad (cuyos determinantes facilitaran o se interpondrán en el manejo de la salud de la persona). La Medicina Familiar cambia el punto de partida del análisis clínico considerando a la persona en su contexto. ${ }^{1}$

\section{Los contenidos de la Medicina Familiar en el Grado}

La MFC debería dar contenidos referidos a la Historia del desarrollo de la especialidad, comprendiendo las necesidades y el contexto social en el que se desarrollo.

Es necesario que los contenidos se desarrollen a cargo de un grupo interdisciplinario, donde se debe desplegar un contenido teórico práctico:

- Inicialmente debe conocer el proceso sociológico y antropológico relevante a nivel mundial y a nivel nacional, para tener un enfoque biopsicosocial integral del ser humano. ${ }^{2}$ 
- conocerá también los enfoques para la determinación de social de la salud y de gestión social del riesgo.

- Profundización en salud ambiental para un enfoque ecológico.

- Conocimiento y profundización en el cuerpo de conocimiento propio de la MFC (problemas de salud más prevalentes y/o graves) que permite elevar la resolución del 90\% de los problemas atendidos en APS.

- Desarrollo de la participación comunitaria en los cuidados de la salud, junto con herramientas que permitan un diagnostico social.

- Fomentar el trabajo interdisciplinario con las otras áreas de la salud. Se pueden crear espacios de trabajo curricular en común para las carreras afines (enfermería, kinesiología, trabajo social, psicología, farmacéuticos, antropólogos, etc).

- Prevención cuaternaria.

- Toma de decisiones basada en evidencias.

- Abordaje integral de la salud de la persona, como actividad de salud eficaz y eficiente que permite abordar el complejo de la salud de la persona y como alternativa al abordaje fragmentado, descoordinado e ineficiente.

- Abordaje de la salud de la familia, conociendo el contexto en el que vive la persona (más que hacer una relación de los antecedentes); conocer los riesgos y problemas de salud que comparten; y proponer y concertar cuidados de salud para superarlos.

Los contenidos deberían revisarse y actualizarse según las necesidades que los sistemas de salud poseen en los diferentes países donde se tenga la posibilidad de un grado en medicina familiar. Los contenidos de la MFC tienen que ser dinámicos y adaptables a las necesidades de cada país.

\section{Escenarios y metodología para el desarrollo de esas competencias clínicas en el Pregrado}

El escenario ideal son los Centros de Atención Primaria (CAPS), implementados o en convenios con las Universidades, con recursos compartidos para la atención de salud y la docencia. En cuanto a la metodología, al menos se debe usar tutoría clínica, aunque también se pueden usar clases expositivas, seminarios de grupos pequeños, aprendizaje basado en problemas, mapas conceptuales, observaciones, simulaciones clínicas. Basándonos en los contenidos de grado los sitios de práctica deben ir encaminados al desarrollo comunitario donde el estudiante podrá tener seguimiento de la comunidad durante su estudio. Realizará un acercamiento a la persona para conocer su historia de vida. Posteriormente, analizará su entorno con enfoque social, luego con las herramientas en medicina familiar. El estudiante será capaz de realizar un enfoque sistémico integral tanto individual, familiar como comunitario. Para que esto sea posible se necesita una fuerte interacción entre la Universidad y el Sector Sanitario.

En el aula se pueden empezar a abordar los conceptos básicos con una metodología educativa de tipo participativo. Esto fomentará la reflexión y el análisis de los alumnos, mediante la utilización de casos clínicos reales. Serían ideales escenarios reales de práctica interdisciplinaria. En este punto se puede considerar espacios curriculares integradores, comunes para varias carreras de las ciencias de la salud, mediante el método de enseñanza basado en problemas.

También se puede llevar a cabo en la consulta clínica con MFC donde tendrán contacto directo con los pacientes, sus principales problemas de salud y el enfoque centrado en la persona y orientado a la familia.

El desarrollo de tutorías presenciales y a distancia que tienen la finalidad de promover la reflexión crítica y el pensamiento complejo. La propuesta metodológica contempla la realización de actividades grupales e individuales de resolución de problemas. Esta metodología propone rescatar los conocimientos previos de los educandos, desde una perspectiva constructivista del proceso de enseñanza-aprendizaje.

\section{La formación de docentes de MFC}

El docente de MFC debe estar formado en un enfoque por competencias que contribuya con la formación de educandos que desafíen problemas del contexto. La integración de saberes (cognitivos, procedimentales y actitudinales) les permitan identificarlos, argumentarlos y resolverlos. Se debiera lograr en los docentes de Medicina 
Familiar, una elaboración conceptual y metodológica que posibilite la observación, análisis, comprensión y diseño de intervenciones significativas en la comunidad.

El grado y la posgrado deberán compartir recursos para tener unidad, congruencia y concordancia en la construcción del conocimiento; y para hacer sostenible la administración de los procesos de enseñanza y eficientes la gestión de la enseñanza. La formación del docente de Medicina Familiar debe ser lo suficientemente amplia como para desplegar habilidades en la enseñanza de diferentes marcos de conocimiento, uno más amplio (formación especializada) y otro más específico (grado), pero la formación es la misma.

La formación de los docentes debe ser continuada, recertificada y obligatoria. De esta manera, se debe presentar una posibilidad para que los profesores puedan optar por una actualización que garantice una educación de calidad. Es necesario que tengan una titulación universitaria en educación. Desde este punto de vista, las habilidades que un docente debe de desarrollar son: el compromiso personal para estar actualizado y la capacidad de adaptación a diferentes ambientes y escenarios. Los docentes que trabajen en el área de pregrado deben realizar un trabajo en equipo con enfoque interdisciplinario donde puedan trabajar junto con enfermeras, antropólogos, médicos de salud pública, gestores de salud, entre otros. El docente debe conocer cómo realizar un tutorial clínico y cómo se debe realizar una devolución al alumno. También deberá entrenarse sobre pautas de observación/evaluación, métodos de evaluación, realización de preguntas escritas y coordinación curricular. Deben tener formación básica docente (aspectos didácticos y pedagógicos), habilidades en nuevas técnicas didácticas y actualización continua específica en temas de APS y MFC.

Al considerar que el público objetivo está en el pregrado, el docente debe estar preparado para utilizar técnicas de enseñanza para jóvenes o adultos, capaz de utilizar los medios tecnológicos y de información/comunicación que manejan estos grupos. Es importante el manejo de la metodología de problematización, de forma que el aprendizaje se genere en consideraciónal conocimiento previo del estudiante, con metodología de diálogo y construcción de conocimiento. Sería favorable que el docente comparta actividades clínicas en MFC.

Es muy importante que, sepa hacer análisis del tipo de estudiantes que tiene, cuáles son sus estilos de aprendizaje.

Los docentes de MFC requieren ser formados interdisciplinariamente en proyectos educativos que se desarrollen en determinado contexto de acción (Espacio Comunitario de Cuidado). En ellos se deben discutir, analizar y compartir las experiencias previas que permitan aprendizajes significativos, relevantes y con sentido desde una mirada integral. Debe promoverse la participación ciudadana activa como un derecho que permita incidir en el proceso de la determinación social de la salud.

\section{Los Contenidos de MFC en el Posgrado (Residencia o Especialización)}

El contenido del Programa de formación de especialistas en MFC podría esquematizarse de la siguiente manera:

a) Cuidado del paciente: el médico en proceso de especialización debe ser capaz de proveer atención al paciente en forma compasiva, apropiada y efectiva para la solución de sus problemas de salud y promoción de la misma. Proveerá los cuidados clínicos necesarios para los pacientes en el entorno ambulatorio y hospitalario en relación con los procedimientos técnicos exclusivos del médico de familia, de acuerdo a la necesidad de la comunidad en donde se desempeñe. Comprenderá la sistemática de atención del individuo sano. Priorizará la prevención en la atención. Ofrecerá continuidad de la atención individual: considerándola como una prioridad mediante la Historia Clínica Orientada al Problema, con información clara y precisa que permita lograr la continuidad de la responsabilidad. Incluirá en la atención pacientes que requieran la continuidad de cuidados en el hogar. Ofrecerá continuidad de los cuidados de los pacientes institucionalizados. Realizará visitas domiciliarias de pacientes adultos mayores, discapacitados, terminales y de riesgo social. Coordinará e integrará la atención de cada paciente, para mantener la continuidad de la responsabilidad de sus pacientes en todos los ámbitos de atención. Será competente en el cuidado de los pacientes de variados orígenes étnicos. ${ }^{3,4}$ 
b) Conocimiento médico: Manifestará su conocimiento acerca de conceptos establecidos y en evolución de las ciencias biomédicas, clínica, epidemiológica, social y ciencias del comportamiento, aplicándolos a la atención del paciente, la familia y la comunidad. Aplicará un pensamiento analítico e inquisitivo en el abordaje de: grupos poblacionales la salud del niño y del adolescente, salud de la mujer, salud del adulto y adulto mayor, salud mental y ciencias del comportamiento humano, medicina comunitaria y gestión de los sistemas de salud. Conocerá y aplicará las ciencias básicas y de soporte relacionadas con la MFC y aplicará principios de metodología de investigación comunitaria, con los diagnósticos de situación, aplicando técnicas de intervención y participación.

c) Aprendizaje basado en la práctica: demostrará la capacidad para investigar y evaluar la atención del paciente y su familia, profundizando y analizando la evidencia científica. Establecerá objetivos de aprendizaje y mejoría de su práctica. Analizará sistemáticamente la práctica utilizando métodos de mejora de calidad. Hallará, evaluará y asimilará evidencia de estudios científicos relacionados con los problemas de salud de los pacientes, la familia y la comunidad. Usará tecnología de la información y de esta forma, apoyará su propia educación. Participará en la educación de los pacientes, de la familia, de la comunidad, de médicos residentes, y miembros del equipo de atención primaria. ${ }^{5}$

d) Habilidades de comunicación interpersonales: El médico residente deberá ser capaz de demostrar habilidades de comunicación interpersonales que resulten en el intercambio efectivo de información y en el establecimiento de una relación de equipo con los pacientes, la familia y profesionales de la salud. Internalizará la metodología comunicacional del lenguaje verbal y no verbal. ${ }^{6}$

e) Profesionalismo: El médico residente debe demostrar su compromiso para llevar a cabo responsabilidades profesionales y cumplir principios éticos.: Demostrará compasión, humanismo, integridad y respeto por los demás. Dará respuesta a las necesidades del paciente, la familia y la comunidad superando el propio interés. Respetará la intimidad y la autonomía del paciente y su familia. Demostrará sensibilidad y capacidad de respuesta a poblaciones diversas sin condicionar la diversidad de género, edad, cultura, raza, religión, discapacidad y orientación sexual. Se iniciará en la Docencia Universitaria y volcará a estudiantes la experiencia adquirida.

f) Gestión de servicios de salud: El médico residente deberá demostrar una toma de conciencia y capacidad de respuesta al contexto mayor del sistema de salud siendo capaz de reclamar en forma selectiva los recursos del sistema para proporcionar una óptima atención. Trabajará de manera eficaz en la atención de la salud en los distintos ámbitos de actuación (hospital, centro de salud, domicilio etc.). Coordinará la atención del paciente y su familia dentro del sistema. Practicará la atención médica teniendo en cuenta la relación de costo efectividad y la distribución de recurso de tal manera que no comprometa la calidad de la misma y la equidad. Sabrá como asociarse con los gerent y proveedores de atención de salud para evaluar, coordinar y mejorar la atención conociendo como estas actividades pueden afectar el funcionamiento del sistema. Sabrá aplicar modelos prestacionales de APS. Incorporará el concepto de accesibilidad y disponibilidad.

g) Enfoque familiar: El objeto de estudio debe ser la familia integrando una serie de habilidades propias del manejo con Familias. La familia como objeto de estudio del médico familiar de incluir la comprensión de la familia como institución social. Reconociendo tipologías familiares, el ciclo evolutivo Individual, de la pareja y familiar, la funcionalidad y disfuncionalidad familiar. Debe utilizar herramientas como el Genograma y enfoques concepctuales tales como la Teoría general de sistemas, Teoría general de la comunicación. Debe adquirir habilidades para la toma de decisiones ante eventos familiares críticos. Deberá entrenarse en intervención del los diversos niveles de prevención información, consejería, orientación y terapia familiar; Tener habilidades para intervenir con otras disciplinas frente a problemas de pareja, violencia intrafamiliar, adicciones, bajo rendimiento escolar, migración, o cualquier situación que altere el bienestar biopsicosocial del grupo. ${ }^{7}$ 
Los escenarios y la metodología para el desarrollo de habilidades clínicas en MFC en el Posgrado (Residencia o Especialización)

Principalmente debe desarrollarse en los CAP, la comunidad, los hogares de los pacientes y en general, todos aquellos escenarios que les permitan estar en contacto directo con los pacientes y puedan evaluar su patología.

Se propone trabajar con metodologías que faciliten la significación y reorganización de conocimientos, valores, actitudes y destrezas que posibiliten el abordaje integral de los problemas de salud en el escenario de la propia comunidad. Se deberán priorizar metodologías innovadoras cuyo objetivo sea el de generar progresivamente competencias en las diferentes áreas contempladas en el programa, para lo cual se deberán realizar las actividades interdisciplinarias y acompañarlas de una autorreflexión que rescate los aspectos positivos y los aspectos a mejorar en cada situación problema.

Los MFC deben desenvolverse en diferentes escenarios desde hospitales, clínicas, servicios de emergencias, consultorios; pero además, desarrollar habilidades desde otras formas de asistencia médica como visita domiciliaria, hospitales de día, equipos de trabajo interdisciplinario para el abordaje de múltiples patologías.

\section{Los escenarios y la metodología para la educación permanente en servicio de los especialistas en Medicina Familiar y Comunitaria}

La Educación Permanente en Salud (EPS) es una estrategia y metodología educativa, impulsada desde 1995 por la Organización Panamericana de la Salud, para el desarrollo del recurso humano y de las capacidades institucionales que permitan mejorar la calidad del cuidado de la salud. La Lic. María Cristina Davini la define como: "Una estrategia de intervención pedagógica-institucional que está orientada hacia el logro de la eficiencia y la eficacia en la prestación de servicios de salud (calidad de la atención y satisfacción de los usuarios), hacia la mejoría de los procesos de trabajo del equipo de salud, y hacia la promoción individual y colectiva de los trabajadores." De acuerdo a este modelo, los MFC refuerzan la integración de las prácticas interdisciplinarias. ${ }^{1,8}$

De este modo, la EPS propone insertar la educación del personal de salud al interior de los equipos de trabajo. Se reúnen para analizar y resolver los problemas del quehacer cotidiano con el objeto de mejorar actitudes, conocimientos y habilidades. ${ }^{9}$

Esta metodología se desarrolla en el lugar de trabajo, analiza la misión institucional del equipo de trabajo y del proceso de trabajo, hace confluir los aspectos técnicos, los procesos humanos y relacionales, los procesos institucionales y el usuario. Está centrada fundamentalmente en la persona, tanto del funcionario como del usuario. Identifica y analiza, en el contexto pertinente, el concepto de Red Asistencial. Los escenarios deberían ser de cada área de salud y debería conducir a los procesos de certificación y recertificación de la competencia profesional. ${ }^{10}$

\section{Evaluaciones cualitativa de los expertos acerca del desarrollo de la Educación en MFC en la región}

Se observó una amplia variabilidad en la percepción de desarrollo de las dimensiones estudiadas, y se observa el esfuerzo que se debe realizar en la Región para mejorar estos aspectos. (Cuadro 1) (Gráficos 1, 2 y 3)

Cuadro 1. Calificación media de dimensiones evaluadas.

\begin{tabular}{|lc|}
\hline Dimensión & Media \\
\hline Desarrollo de la Medicina Familiar en Universidad & 5,6 \\
Formación de los Docentes en Medicina Familiar & 5 \\
Desarrollo Certificación de la Especialidad & 5,2 \\
\hline
\end{tabular}


Evaluación del desarrollo de la medicina familiar en la

Educación Universitaria de Grado en el pais

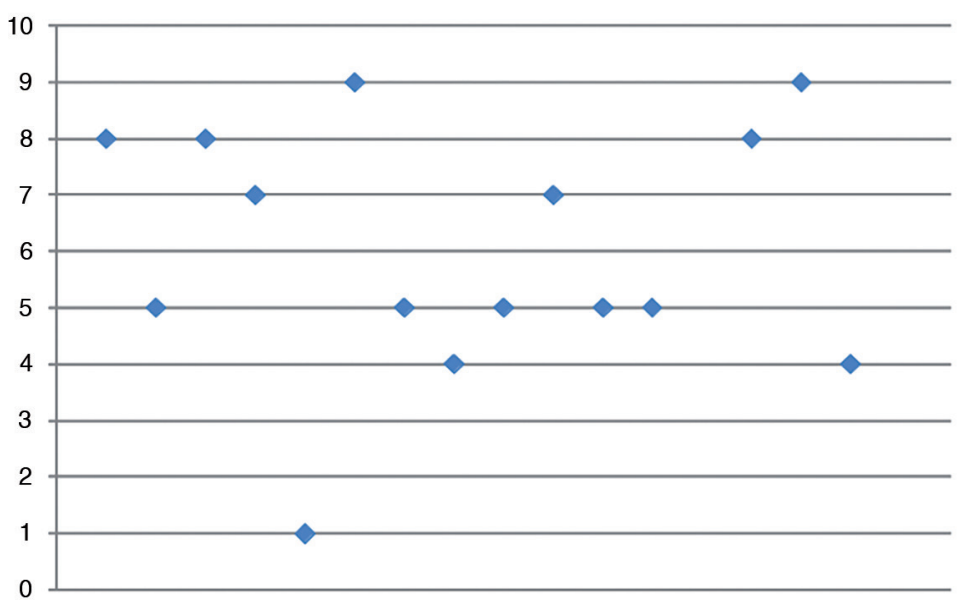

Grafico 1. Evaluación del desarrollo de la medicina familiar en el pais.

Calificación de la formación de docentes de Medicina

Familiar en el pais

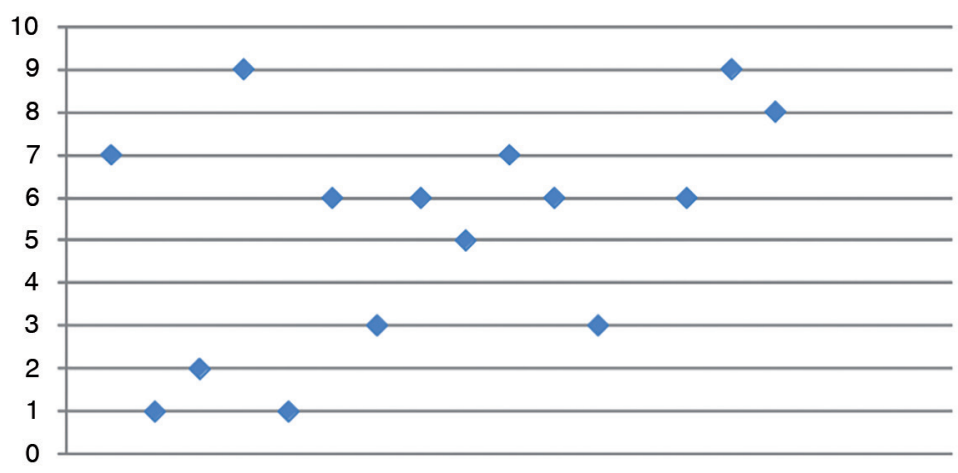

Grafico 2. Calificación de la formación de docentes de Medicina Familiar en el país.

Estado de desarrollo de los procesos de certificación de la especialidad de Medicina Familiar en el pais

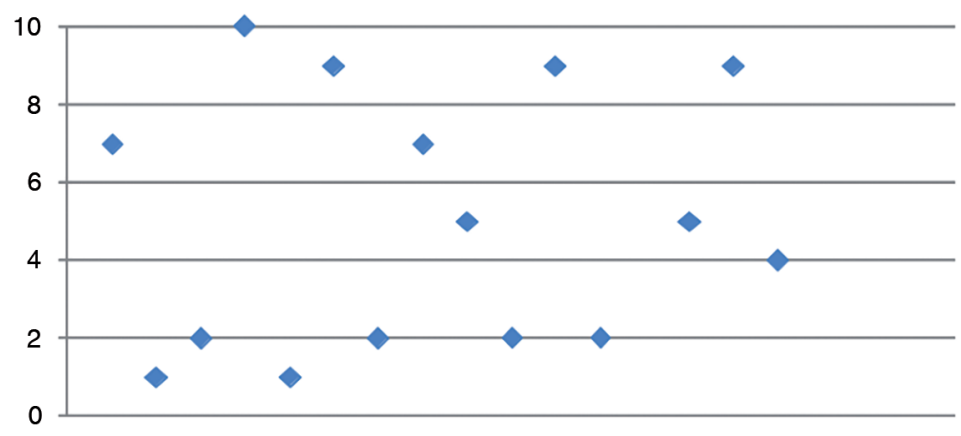

Grafico 3. Estado de desarrollo de los procesos de certificiacion de la especialidad de medicina familiar en el pais. 


\section{Discusión}

Realizar un abordaje integral del Proceso Salud Enfermedad Atención requiere, en primer lugar, repensar nuestra propia práctica y comprender que este "modo de hacer" se sustenta en una determinada conceptualización de la salud y su cuidado, en cierta noción de la persona y su entorno familiar y comunitario y en determinada forma de construcción de los saberes y las prácticas de los equipos de salud. ${ }^{2,3}$

En un PEA, a fin de hacer entendible la complejidad del Proceso Salud Enfermedad - Atención, deben procurarse dispositivos de comprensión y abordaje que pongan en evidencia la relación entre los procesos generales de la sociedad, los diferentes modos de vida de los grupos y las particulares condiciones de los estilos de vida de las personas. ${ }^{4}$

Debemos partir, entonces, del concepto de enfermedad como un proceso que no resulta de la acción externa de un agente ambiental agresivo, ni de la reacción internalizada de un huésped susceptible, sino de un proceso totalizador de efectos patológicos, y comprender a la salud como un continuo en permanente tensión y conflicto en la búsqueda de una mejor calidad de vida. Este proceso está condicionado por las potencialidades, capacidades y limitaciones que las personas, las familias y las comunidades evidencian en el manejo de los recursos disponibles. ${ }^{8,9}$

\section{Conclusiones}

1. Los contenidos para la formación están orientados a promover la adquisición de competencias profesionales que faciliten el desarrollo de un modelo integral y holístico de abordaje del proceso salud-enfermedadatención sustentado en un enfoque de determinación social que propicie la resolución de los problemas de salud de mayor prevalencia en nuestras poblaciones, en todas las etapas del ciclo vital.

2. La incorporación de las propuestas educativas de Medicina Familiar y Comunitaria requiere una reflexión profunda, que considere entre otros aspectos, las amenazas provenientes de las políticas educativas, el sistema económico, los movimientos sociales, los estilos de administración y las culturas organizacionales que prevalecen en nuestras Universidades sustentadas en su mayoría en el paradigma biomédico.

3. El escenario ideal de formación es el de un Centro de Atención Primaria de la Salud. En cuanto a la metodología, se debería usar principalmente la tutoría, aunque también se pueden usar otras metodologías como: seguimiento en consultorio de MFC, talleres en el aula, juegos de rol y cámara de Gessell, Videograbaciones del alumno en la consulta médica, Aprendizaje Basado en Problemas y Método de Casos.

\section{Referencias}

1. Jure H, Acuña M, Coppolillo F, Ciuffolini, Didoni M. Cuadernos de Salud Familiar y Comunitaria. Buenos Aires. 2011.

2. Almeida Filho N. La Ciencia Tímida, Ensayos de deconstrucción de la epidemiología. Buenos Aires: Editorial Lugar; 2000.

3. Breilh J. Epidemiología Critica. Ciencia Emancipadora e Interculturalidad. Buenos Aires: Editoral Lugar; 2003.

4. Ciuffolini MB, Jure H. "Estrategias de comprensión integral del proceso salud- enfermedad: aportes desde la perspectiva de la vivienda saludable". Astrolabio. Centro de Estudios Avanzados. UNC. № 3. Noviembre 2006.

5. Kuhn TS. La estructura de las revoluciones científicas. Buenos Aires: Fondo de Cultura Económica; 1998.

6. Mc Whinney Ian. Medicina de Familia. Madrid: Ed. Doyma; 1995.

7. Menéndez E. "Modelos de atención de los padecimientos: de exclusiones teóricas y articulaciones prácticas”. Salud Colectiva. Spinelli Hugo, Compilador. Buenos Aires: Editorial Lugar; 2005:11-47.

8. Morin E. Introducción al pensamiento Complejo. Editorial Gedisa: Barcelona;2005.

9. Rojas MC, Ciuffolini MB, Meichtry N. "La vivienda saludable como estrategia para la promoción de la salud en el marco de la medicina familiar" Archivos de Medicina Familiar. Editorial Medicina Familiar Mexicana. 2005;7(1):27-30.

10. Sousa Campos G. Gestión en Salud, en Defensa de la Vida. Buenos Aires: Editorial Lugar; 2001. 